\title{
Design and Realization of ADC based on FPGA Jing $\mathrm{Wu}$

\author{
School of Intelligence Science and Information Engineering, \\ Xi'an Peihua University, Xi'an, China \\ 448737641@qq.com
}

Keywords: Analog to digital controller; Central processing unit; Field programmable gate array

\begin{abstract}
In this paper, the signal collected by WM8731 that is a high resolution analog to digital controller (ADC) is set to establish custom NIOS II central processing unit (CPU) and peripheral controlling in the system on a programmable chip (SOPC) Builder, systems developing tool, of Altera Quartus II that is a developing software. The design and realization of ADC based on FPGA is designed, ran, compiled and simulated in Quartus II. The Design and Realization of ADC Based on field programmable gate array (FPGA) combines the advantages of embedded system and FPGA and provides a new design method of digital signal processing system of high performance. The improved method is proved to be effective.
\end{abstract}

\section{Introduction}

With the development of microelectronic technology, the design of electronic system is design of SOPC based on large scale FPGA instead of using various integrated circuit(IC) to carry out printed circuit board (PCB) board. At the same time, the rapid development of electronic design automatic (EDA) tools is also makes it is possible to realize the design of the whole system from the behavior algorithm level to the physical structure level implemented on FPGA[1]. And Quartus II and NIOS II introduced by Altera company provide great convenience for realizing the SOPC system based on embedded IP core of FPGA.

In this paper, the signal collected by WM8731 that is a high resolution analog to ADC is set to establish custom NIOS II CPU and peripheral controlling in the system on SOPC Builder, systems developing tool, of Altera Quartus II which is a developing software. The design and realization of ADC Based on FPGA is designed, ran, compiled and simulated in Quartus II.

\section{Design and Realization of ADC based on FPGA}

The signal collected by WM8731 is set to establish custom NIOS II CPU and peripheral controlling in the system on SOPC Builder based on FPGA.

The overall system block diagram of ADC based on FPGA is shown in Fig. 1.

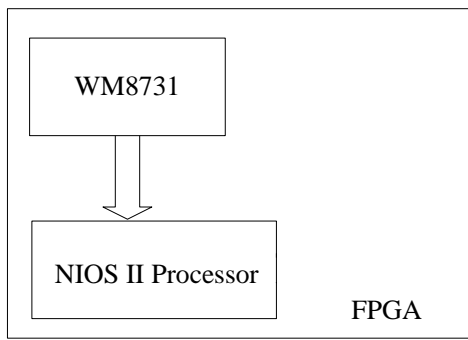

Figure 1. The overall system block diagram

\subsection{Design of Hardware of WM8731 Based on FPGA.}

The general ADC has 8bits, 10bits, 12 bits, 14 bits and 16 bits. In order to obtain good linearity and high resolution of the instrument, ADC with 12 bits to 16 bits are required to collect the data of signal[2]. WM8731 supports digital audio signal from16 bits to 32 bits and realizes sampling rate 
range 8 kilohertz to 96 kilohertz. It can directly convert the very weak input[3] signal received from the sensor into a serial digital signal without an external instrument amplifier. The WM8731 with 16 bit is used in this paper.

Design of hardware of WM8731 based on FPGA. The digital audio interface of WM8731 is the input and output interface. I2S mode, right justified mode, left justified mode and DSP mode are supported by the transmission of serial data. These modes are selected by word control. By selecting transmission mode, WM8731 is permitted to work in normal mode. The complete description of VHDL[4] is omitted according to the timing of WM8731 operating in normal mode.

Simulation of WM8731. In Quartus II [5] it is designed, compiled and emulated.

Simulation waveform of WM8731 is shown in in Fig. 2.

\begin{tabular}{|c|c|c|c|c|c|c|c|c|c|}
\hline \multirow[b]{2}{*}{ ame } & $0 \mathrm{ps}$ & $80.0 \mathrm{~ns}$ & $160.0 \mathrm{~ns}$ & $240.0 \mathrm{~ns}$ & $320.0 \mathrm{~ns}$ & $400.0 \mathrm{~ns}$ & $480.0 \mathrm{~ns}$ & $560.0 \mathrm{~ns}$ & $640.0 \mathrm{n}$ \\
\hline & $0 \mathrm{ps}$ & & & & & & & & \\
\hline BCLK & $\begin{array}{l}\square \quad \\
\end{array}$ & L & 5 & 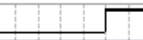 & $2 \quad$ & 4 & & 5 & $\square \quad 5$ \\
\hline 田 ADCLRC & $\square \quad$ & \begin{tabular}{l|l|} 
& -12 \\
\end{tabular} & +1 & \begin{tabular}{l|l}
+1 & 1 \\
\end{tabular} & $\begin{array}{lll}22 & \\
\end{array}$ & 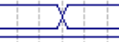 & -5 & 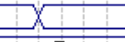 & -27 \\
\hline 田 ADCDATA & 0 & K & -12 & $\quad x$ & \begin{tabular}{l:l|l} 
& & 1 \\
\end{tabular} & 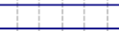 & $x$ & -5 & +1 \\
\hline
\end{tabular}

Figure 2. Simulation waveform of WM8731

The VHDL code of WM8731 is compiled and simulated in Quartus II. The simulation waveform is shown in figure 5 . The results show that when the BCLK is high, the converted data, $-12,22,-5$, are output by ADCDATA. The simulation results show that the controlling conversion and output can be realized well.

The encapsulated Module Diagram of WM8731.The encapsulated module diagram of WM8731 is shown in Fig. 3.

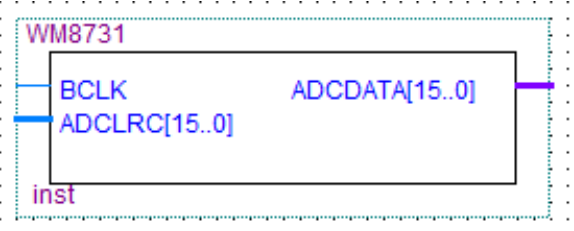

Figure 3. The encapsulated module diagram of WM8731

\subsection{Design and Realization of NIOS II CPU Based on FPGA.}

In this paper, the custom NIOS II central processing unit (CPU) and peripheral are established by the system on a programmable chip (SOPC) Builder, systems developing tool, of Altera Quartus II, developing software, and meet the requirements of the processor system. SOPC Builder configures embedded processing chip of high integrated SOPC system by loading NIOS II core and peripheral interfaces. NIOS II processor is a complete software development suite, including a compiler. Integrated development environment (IDE), JTAG debugger, real-time operating system (RTOS) and TCP/IP protocol stack and so on.

The hardware design flowchart of NIOS II CPU. Using powerful system developing tool of SOPC Builder of Altera and the design software, Quartus II and NIOS IDE, the design of NIOS II CPU can be completed.

The flow chart of NIOS II CPU is shown in Fig. 4. 


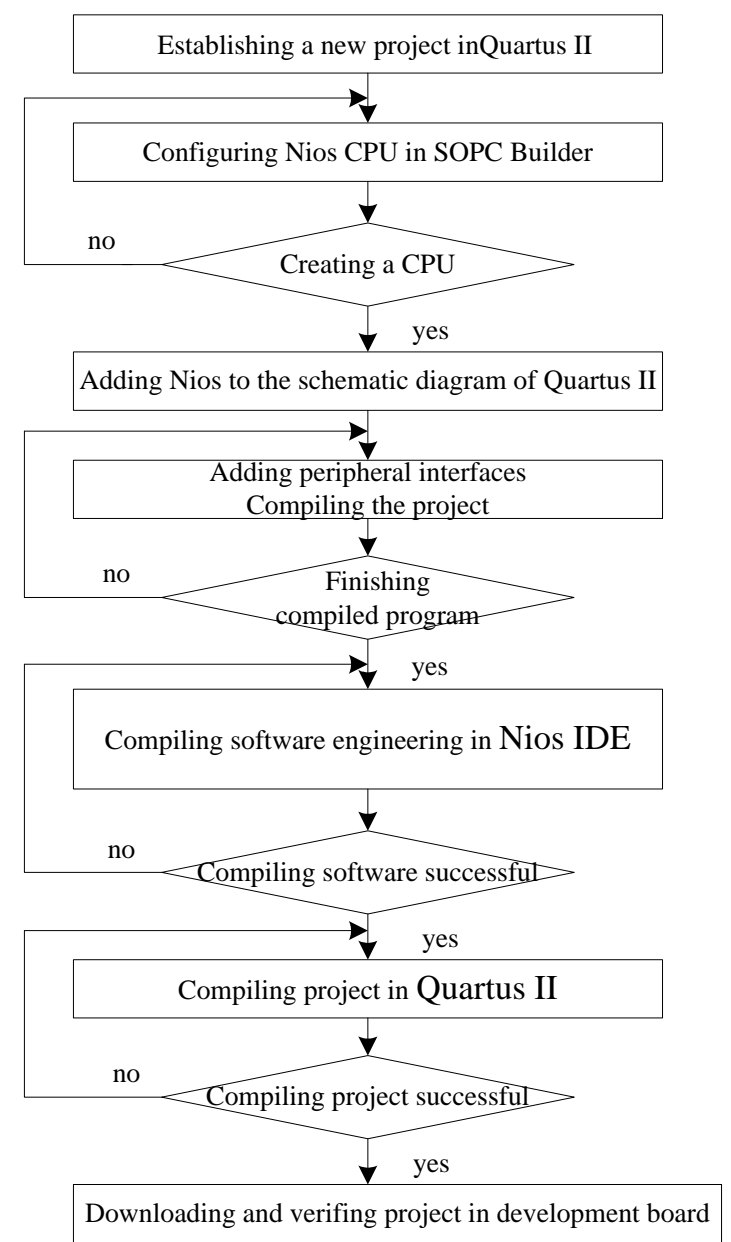

Figure 4. The hardware Design flow chart of NIOS II CPU

The design of hardware of NIOS II CPU based on FPGA. The design of hardware of NIOS II is consist of serial peripheral interface PIO, serial UART, timer, SDRAM external memory, SRAM and Flash external memory. The hardware design of NIOS II CPU mainly includes graphical user interface (GUI) and program generated by system. PTF files that describe the system are created by GUI. Each component of GUI can also provide its own configuration graphical user interface. The program generated by system generates a description of HDL for the target device. Given the limited space, the program generated by system is not listed here.

The graphical user interface is shown in Fig. 5.

\begin{tabular}{|c|c|c|c|c|c|c|c|c|}
\hline Use & $\ldots$ & Module Name & Description & Clock & & ase & End & $\mathbb{R Q} Q$ \\
\hline$\nabla$ & & $\boxplus$ cpu & Nios II Processor & sys_clk & m & $0 \times 01903000$ & $0 \mathrm{z} 019037 \mathrm{ff}$ & \\
\hline$\square$ & & 田 ext_ram_bus & Avalon-MM Tristate Bridge & sys_clk & & & & \\
\hline$\sqrt{\square}$ & & 田 ext_flash & Flash Memory (CFI) & sys_clk & in & $0 \times 01400000$ & $0 \mathbf{z} 017 \mathrm{fffff}$ & \\
\hline$\square$ & & 田 sdram & SDRAM Controller & sys_clk & in & $0 \times 00800000$ & $0 z 00 f f f f f$ & \\
\hline$\square$ & & 田 onchip_mem & On-Chip Memory (RAM or ROM) & sys_clk & if & $0 \times 01901000$ & $0 \mathrm{z} 01901 \mathrm{fff}$ & \\
\hline$\square$ & & 田 jtag_uart & JTAG UART & sys_clk & 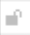 & $0 \times 01904120$ & $0 \mathbf{2 0 1 9 0 4 1 2 7}$ & \\
\hline$\square$ & & 田 LED_GREEN & PIO (Parallel INO) & sys_clk & wi & $0 \times 01904080$ & $0 \mathrm{z} 0190408 \mathrm{f}$ & \\
\hline$\square$ & & 田 LED_RED & PIO (Parallel I/O) & sys_clk & If & $0 \times 01904090$ & $0 \mathbf{z} 0190409 f$ & \\
\hline$\square$ & & 田 BUTTON & PIO (Parallel INO) & sys_clk & if & $0 \times 019040 a 0$ & $0 \mathrm{z} 019040 \mathrm{af}$ & \\
\hline$\square$ & & 田 SWITCH & PIO (Parallel I/O) & sys_clk & $=$ & $0 \times 019040 b 0$ & $0 \mathrm{z} 019040 \mathrm{bf}$ & \\
\hline$\square$ & & 田 sys_clk_timer & Interval Timer & sys_clk & if & $0 \times 01904000$ & $0 \mathrm{z} 0190401 \mathrm{f}$ & \\
\hline$\square$ & & 田 high_res_timer & Interval Timer & sys_clk & min & $0 \times 01904020$ & $0 z 0190403 f$ & \\
\hline$\square$ & & 田 sysid & System ID Peripheral & sys_clk & fif & $0 \times 01904128$ & $0 \mathbf{0} 0190412 \mathrm{f}$ & \\
\hline$\square$ & & 田 epcs_flash_controller & EPCS Serial Flash Controller & sys_clk & fin & $0 \times 01903800$ & $0 \mathrm{z} 01903 \mathrm{fff}$ & \\
\hline$\square$ & & 田 pll & PLL & clk & fir & $0 \times 01904040$ & $0 z 0190405 f$ & \\
\hline$\square$ & & 田 Icd & Character LCD & sys_clk & If & $0 \times 019040 c 0$ & $0 \mathrm{z} 019040 \mathrm{cf}$ & \\
\hline$\square$ & & 田 uart & UART (RS-232 Serial Port) & sys_clk & m & $0 \times 01904060$ & $0 \geq 0190407 f$ & \\
\hline$\square$ & & $\boxplus$ isp1362 & PIO (Parallel INO) & sys_clk & (in & $0 \times 00000000$ & $0 \mathbf{z} 0000000 \mathrm{f}$ & \\
\hline$\square$ & & 田 ADCINT & PIO (Parallel I/O) & sys_clk & min & $0 \times 01904100$ & $0 \mathrm{z} 0190410 \mathrm{f}$ & \\
\hline$\square$ & & 由 AUDAT_IN & PIO (Parallel IO) & sys_clk & min & $0 \times 01904110$ & $0 \mathbf{0} 0190411 \mathrm{f}$ & \\
\hline
\end{tabular}

Figure 5. The graphical user interface 
Realization of NIOS II CPU based on FPGA. The hardware circuit of NIOS II is big and leaved out. Its input ports are described simply as follows.

clk: in std_logic;

Reset_n: in std_logic;

In_port_to_the_ADCINT: in std_logic;

In_port_to_the_AUDAT_IN: in std_logic_vector(15 downto 0);

In_port_to_the_BUTTON: in std_logic_vector(2 downto 0);

\section{Design and Realization of ADC Based on FPGA}

Using schematic input design method, the completed WM8731 and NIOS II CPU are taken as components of bottom design and encapsulated, then the top design, the design and realization of ADC based on FPGA, is finished in the edit window in Quartus II . It is realized as follow:

u1:WM8731 PORT MAP (BCLK=>clk,ADCDATA=>in_port_to_the_AUDAT_IN);

u2: NIOS II 81 PORT MAP (clk => BCLK, in_port_to_the_AUDAT_IN => ADCDATA);

Design and Realization of ADC Based on FPGA is well finished by Schematic design input method. As the hardware circuit of ADC Based on FPGA is too big, it can not be presented clearly and is omitted .

\section{Conclusions}

Quartus II provides a complete multi-platform design environment and is a comprehensive system design environment. Quartus II combining with SOPC Builder and NIOS II IDE provides a complete SOPC solution, makes designer to develop system conveniently and reflects the outstanding advantages of EDA tools in the designing system. The improved method is proved to be effective.

\section{Acknowledgment}

This work is supported by the Shaanxi Province Science Foundation under Grant No. 17JK1056.

\section{References}

[1] Yinghui. 2017.Development Status and Prospect of Deformation Monitoring Techniques for High-Speed Rails, 2017 2nd International Conference on Machinery,Electronics and Control Simulation(MECS 2017), 138, 908-912.

[2] Peng Zhao; Fan Li; Jianhui Zhao; Jin Ding.2016. Design and Simulation of Low Noise Amplifier in Lower Frequency, 2016 4th International Conference on Machinery,Materials and Information Technology Applications(ICMMITA 2016), 71, 474-477.

[3]Thibault Barbié; Takeshi Nishida. 2017.Trajectory Prediction using Conditional Generative Adversarial Network,2017 International Seminar on Artificial Intelligence, Networking and Information Technology, 193-197.

[4] Juan Wang; Shengli Hou; Shengquan Zhang. 2016.Development of Full-waveform Acquisition Device in High Power Electrical Transmitter,2016 International Conference on Computer Engineering, Information Science \& Application Technology(ICCIA 2016), 499-503.

[5] Li-hua JIANG1; China Ya-qin Li. 2015. Design and Realization of Multi-port Register File based on Schematic,2010 Third International Symposium on Knowledge Acquisition and Modeling (KAM 2010), 1373-1376. 